\title{
Narrow-host-range IncP Plasmid pHH502-1 Lacks a Complete IncP Replication System
}

\author{
By CHRISTOPHER A. SMITH AND CHRISTOPHER M. THOMAS* \\ Department of Genetics, University of Birmingham, PO Box 363, Birmingham B15 2TT, UK
}

(Received 23 February 1987; revised 13 April 1987)

\begin{abstract}
Plasmid pHH502-1 shows incompatibility only towards members of the IncP group, but has a narrower host range than typical members of that group. In contrast to other IncP plasmids its replication was not affected by a high-copy-number plasmid carrying the replication origin (oriV) of IncP plasmid RK2. Southern blotting of pHH502-1 revealed homology to oriV, consistent with its incompatibility phenotype, but no homology to $\operatorname{tr} f A$, the essential replication gene of RK2. Thus it is probable that pHH502-1 does not possess a functional IncP replication system, accounting for its restricted host range. A restriction map of pHH502-1 was constructed and the mercury-resistance determinant was localized to $\mathrm{Tn} 735$, which also carries the trimethoprim-resistance determinant and is related to $\mathrm{Tn} 21$. The presence of a kor $B$-like function on pHH502-1 was also demonstrated.
\end{abstract}

\section{INTRODUCTION}

Plasmids which cannot be stably maintained together in the same host cell line are said to be incompatible and to belong to the same incompatibility group; such groups often reflect the possession by their members of related replication systems. Although most plasmids belong to only one incompatibility group, some are members of more than one group, being incompatible with members of two or more groups of plasmids, other members of which are incompatible only with the members of their own group. Such plasmids may possess all or part of two or more replication systems.

Typical plasmids of Escherichia coli incompatibility group P (equivalent to the Pseudomonas group IncP-1) have a broad host range, being capable of self-transfer to and maintenance in members of most Gram-negative genera. These plasmids have been divided into two sub-groups which are relatively distantly evolutionarily related: IncP $\alpha$, including the indistinguishable isolates RK2, RP1, RP4, R18 and R68, which are the best-studied members of the IncP group, and IncP $\beta$, including R751, R906 and R772 (Chikami et al., 1985; Smith \& Thomas, 1987). Although the control circuits of RK2 are complex, only two loci are essential for vegetative replication, oriV, the origin of replication, which is closely linked to major determinants of $\mathbf{P}$ group incompatibility, and $\operatorname{tr} f A$, encoding a trans-acting product (Thomas et al., 1985). Incompatibility is also expressed by the region of RK 2 encoding $\operatorname{kor} A$ and $k o r B$. These loci are involved in the regulation both of $\operatorname{trf} A$ and of a number of kil genes which cannot be cloned in their absence (Figurski et al., 1982; Thomas \& Hussain, 1984). Thus the IncP grouping appears to result from the possession of related replication systems by its members.

Certain plasmids are incompatible with IncP plasmids but have much narrower host ranges than the typical members of the group. Examples of IncP plasmids unable to establish themselves in certain hosts when tested for self-transfer include: pMU700-pMU707, in Pseudomonas and Proteus (Grant et al., 1980); pHH502, in Acinetobacter, Proteus, Providencia, Pseudomonas and Serratia, and its derivative pHH502-1, in Acinetobacter and Pseudomonas (Nugent et al., 1982); and pAV1, which is restricted to certain strains of Acinetobacter calcoaceticus (Hinchliffe \& Vivian, 1980). Of these, pMU700-pMU707 and pHH502 are also 
incompatible with IncI $\alpha$ plasmids, while pAV1 has only been tested for incompatibility with plasmids of group P. In contrast, pHH502-1, which arose from pHH502 by a repeatable spontaneous event apparently involving both deletion and rearrangement, lacks IncI $\alpha$ characteristics and does not show incompatibility with members of any other known group (Nugent et al., 1982).

We have investigated pHH502-1, because we are interested in the basis for the broad host range of typical IncP plasmids and hoped that the differences between its replication system and those of other IncP plasmids might reveal features of importance in determining plasmid host range. Here we report evidence that this plasmid both possesses a non-IncP replication system and lacks a complete IncP replication system. We also present a restriction map of pHH502-1 and show that $\mathrm{Tn} 735$ carries the $\mathrm{Hg}^{\mathrm{r}}$ determinant as well as the $\mathrm{Tp}^{\mathrm{r}}$ determinant of the plasmid and that this transposon is related to $\operatorname{Tn} 21$.

\section{METHODS}

Bacterial and plasmid strains. The bacterial host strain used for pHH502-1 was MV10, a trpE5 derivative of $E$. coli $\mathrm{K} 12$ strain $\mathrm{C} 600$ (thr-1 leu-6 thi-1 lac YI supE44 tonA2I). pHH502-1 (Nugent et al., 1982) confers resistance to trimethoprim and to mercuric salts, and low-level resistance to sulphonamides. RK2 (Ingram et al., 1973) confers resistance to penicillin, kanamycin and tetracycline. R772 (Coetzee et al., 1979) confers resistance to kanamycin and carries an inactive mercury-resistance determinant (Smith \& Thomas, 1987). R906 (Hedges et al., 1974) confers resistance to oxacillin, streptomycin, sulphonamides and mercuric salts. R751 (Jobanputra \& Datta, 1974) confers resistance to trimethoprim. References to plasmids constructed by in vitro recombination are given below.

Testing for sensitivity to P-group incompatibility. MV10(pHH502-1) was transformed with the ColE1-derived plasmids pCT7 and pCT8, which both confer resistance to kanamycin and are isogenic except that pCT7 carries the oriV region of RK2 (Thomas et al., 1980). In each case 100 kanamycin-resistant colonies were tested for trimethoprim resistance, indicating retention of pHH502-1, as described for R751 (Smith \& Thomas, 1985).

Testing for KorA and KorB phenotypes. Attempts were made to transform MV10(pRK259.2) and MV10(pHH502-1) with the $\mathrm{KilA}^{+}$plasmid pCAS54, selecting for penicillin resistance, and with the $\mathrm{KilB}^{+}$ plasmid pCAS51, selecting for tetracycline resistance. Transformant colonies are expected only if a functional copy of the $k$ or $A$ or $k o r B$ gene, respectively, is already present in the recipient; both genes are carried by pRK 259.2 (Smith \& Thomas, 1983).

Southern blotting. Isolation of plasmid DNA, restriction analysis, agarose gel electrophoresis, preparation and radio-labelling of probe fragments, transfer of DNA fragments to nylon membranes, hybridization and autoradiography were done as described by Smith \& Thomas (1987). The RK2-derived probe fragments carrying the oriV region and the $\mathrm{C}$-and $\mathrm{N}$-terminal parts of the $\operatorname{trf} A$ gene correspond respectively to fragments 5,6 and 7 of Smith \& Thomas (1987), obtained from EcoRI + HindIII digests of plasmids pCAS220, pCAS226 and pCAS228. The probe fragments derived from $\mathrm{Tn} 501$ were the two internal EcoRI fragments of the transposon, corresponding to fragments 9 and 10 of Smith \& Thomas (1987).

\section{RESULTS AND DISCUSSION}

\section{Restriction map}

We constructed a restriction map of pHH502-1 (Fig. 1), which confers resistance to trimethoprim $\left(\mathrm{Tp}^{r}\right)$ and mercuric ions $\left(\mathrm{Hg}^{r}\right)$ and a partial resistance to sulphonamides, designated $\left[\mathrm{Su}^{r}\right]$ by Nugent et al. (1982). Nugent et al. (1982) reported that three of the EcoRI fragments of pHH502-1, with sizes of $3.2,2.8$ and $0.85 \mathrm{MDa}(4.8,4.2$ and $1.3 \mathrm{~kb})$, are contained within $\mathrm{Tn} 735$, a transposable element of $9.85 \mathrm{MDa}(15 \mathrm{~kb})$ which carries the $\mathrm{Tp}^{\mathrm{r}}$ determinant of the plasmid. These fragments, together with two small EcoRI fragments not previously reported, span coordinates $13.1 \mathrm{~kb}$ to $23.7 \mathrm{~kb}$ of our map. The distribution of cleavage sites on the map of pHH502-1 is non-uniform, with 16 of the 25 sites lying within $\operatorname{Tn} 735$ (Fig. 1). Such clustering of restriction sites in the regions encoding resistance determinants is also found in the case of typical IncP plasmids (Smith \& Thomas, 1987).

\section{Location of mercury-resistance determinant}

In order to locate the mercury-resistance determinant of pHH502-1 we used the two internal EcoRI fragments of the mercury-resistance transposon Tn 501 as probes against Southern blots of digests of the plasmid (Fig. $2 a, b$ ). Each of these fragments carries part of the mercuryresistance operon (mer) of Tn501 (Brown et al., 1983). The regions of overlap between the 


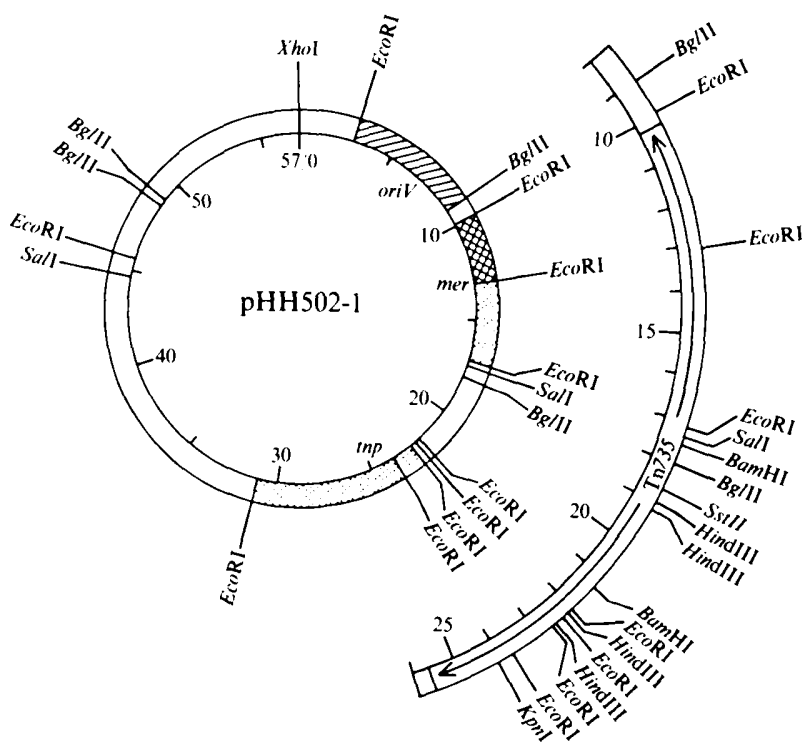

Fig. 1. Restriction map of $\mathrm{pHH} 502-1$. The region between coordinates $8 \mathrm{~kb}$ and $26 \mathrm{~kb}$ is expanded for greater clarity, and the probable end-points of $\mathrm{Tn} 735$ are indicated. The regions containing homology to the probes used are indicated as follows: RK2 oriV by hatching; Tn50I shorter fragment (mer) by cross-hatching; Tn50I larger fragment (mer and $t m p$ ) by stippling.

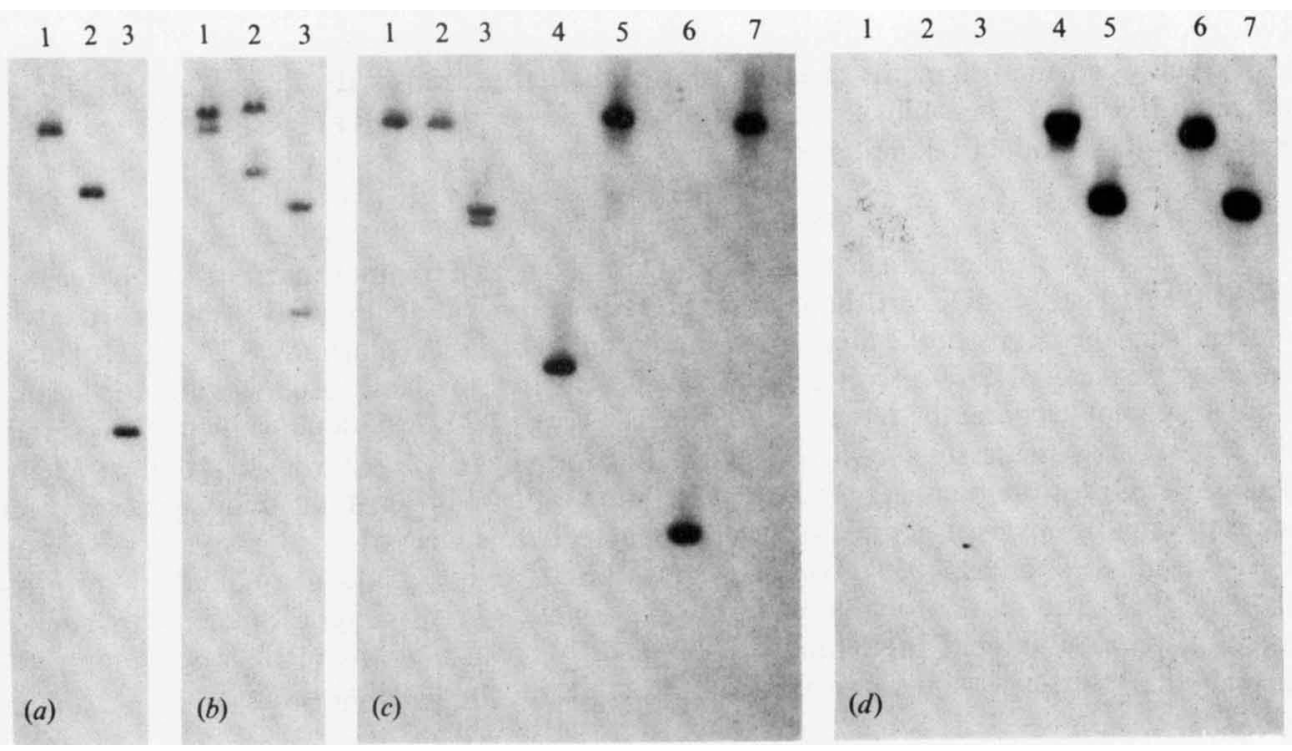

Fig. 2. Southern blots of digests of pHH502-1, R772 and R906. Probe fragments were derived from: (a) and $(b)$ the shorter and longer EcoRI fragments of Tn 501 respectively; $(c)$ the oriV region of $\mathrm{RK} 2 ;(d)$ the $\operatorname{trf} A$ region of RK2. The lanes contained: (1) pHH502-1 digested with $\mathrm{SalI}+\mathrm{Xhol}$; (2) pHH502-1 digested with $B g / I I$; (3) pHH502-1 digested with EcoRI ; (4) R772 digested with Pst I ; (5) R772 digesied with EcoRI ; (6) R906 digested with Sst II ; (7) R906 digested with EcoRI. The sizes (kb) of the fragments in each digest showing homology to each probe were as follows. (1):(a) $17 \cdot 5 ;(b) 27$ and $17 \cdot 5 ;(c) 17 \cdot 5 ;(d)$ none. (2): (a) $9 \cdot 0 ;(b) 31$ and $9.0 ;(c) 17.0 ;(d)$ none. (3): (a) $3 \cdot 3 ;(b) 7.2$ and $4 \cdot 2 ;(c) 6.1$ and $5.9 ;(d)$ none. (4): (c) 3.6; (d) 27. (5): (c) 29; (d) 9.7. (6): (c) 2.2; (d) 21.5. (7): (c) 27.5; (d) 9.7. 
fragments to which each probe hybridized in the digests of pHH502-1 are indicated in Fig. 1. The two probes hybridized to adjacent EcoRI fragments at coordinates $9.9 \mathrm{~kb}$ to $13.1 \mathrm{~kb}$ and $13.1 \mathrm{~kb}$ to $17.2 \mathrm{~kb}$, indicating that the mer genes of the plasmid are in this region, and share a common EcoRI site with those of Tn501. Since one of these EcoRI fragments is internal to Tn735 (Nugent et al., 1982), the transposon must carry the $\mathrm{Hg}^{\mathrm{r}}$ determinant as well as the $\mathrm{Tp}^{\mathrm{r}}$ determinant of pHH502-1.

\section{Relatedness of Tn735 to Tn21}

The larger of the two Tn501-derived probe fragments hybridized to two regions of pHH502-1 (Fig. $2 b$ ): to the mer region and to a region at the other end of Tn735 (Fig. 1). Since this probe fragment contains the transposition (tnp) genes of $\mathrm{Tn} 501$ as well as part of the mer operon (Diver et al., 1983), it appeared possible that the second region of hybridization on pHH502-1 was due to homology between the tnp genes of $\mathrm{Tn} 735$ and $\mathrm{Tn} 501$. This hypothesis was confirmed by the distribution of BamHI, EcoRI and HindIII sites between coordinates $13.3 \mathrm{~kb}$ and $23.7 \mathrm{~kb}$ within $\mathrm{Tn} 735$. This distribution is identical with that found in the $\operatorname{tn} p$ region of $\operatorname{Tn} 21$ (and of several other closely related transposons), to which the thp region of Tn 501 is related (Tanaka et al., 1983: Kratz et al., 1983; Diver et al., 1983). Fig. 1 shows the end-points predicted for Tn735 if its outer ends correspond to those of $\mathrm{Tn} 21$ and its relatives. These end points give a total size of $15.4 \mathrm{~kb}$ for $\mathrm{Tn} 735$, in agreement with the size reported by Nugent et al. (1982). The Tpr determinant carried by Tn 735 presumably lies within the EcoRI fragment between coordinates $17.2 \mathrm{~kb}$ and $22 \mathrm{~kb}$, which does not show homology to Tn501. Although there is no direct evidence concerning the location of the [ $\left.\mathrm{Su}^{\mathrm{r}}\right]$ determinant of $\mathrm{pHH}$ 502-1, it is noteworthy that the distribution of SalI, BamHI, BglII and HindIII sites between coordinates $17.4 \mathrm{~kb}$ and $19 \cdot 2 \mathrm{~kb}$ within $\mathrm{Tn} 735$ is similar to that within the $\mathrm{Su}^{\mathrm{r}}$ determinant of $\mathrm{Tn} 21$ and its relatives (Kratz et al., 1983).

\section{Homology to the oriV region}

Fig. 2(c) shows a Southern blot of digests of pHH502-1 and the IncP $\beta$ plasmids R772 and $\mathrm{R} 906$ probed with a fragment carrying the oriV region of the IncP $\alpha$ plasmid RK2. The region of overlap between the fragments to which this probe hybridized in the digests of pHH502-1 is indicated in Fig. 1. The smaller of the two bands seen in the EcoRI digest is believed to be a partial EcoRI* product of the larger.

\section{Lack of homology to the $\operatorname{trf} A$ gene}

Fig. $2(d)$ shows the same digests as Fig. $2(c)$, probed with a fragment carrying the $\mathrm{N}$-terminal part of the $\operatorname{trf} A$ gene of RK2. Although the autoradiograph was deliberately overexposed (as can be seen from the bands in the lanes containing digests of IncP $\beta$ plasmids R772 and R906), no homology between pHH502-1 and this probe was detected; the same result was obtained using a probe-fragment carrying the remainder of the $\operatorname{trf} A$ gene of RK2 (data not shown). This implies that if pHH502-1 possesses a $\operatorname{tr} f A$-like gene its sequence has diverged from that of the $\operatorname{trf} A$ gene of RK 2 too far for the homology to be detected under the hybridization conditions used. Since under the conditions used homology was easily detected between the oriV regions of RK2 and R751 (Smith \& Thomas, 1987), which show only $65 \%$ overall sequence homology (Smith \& Thomas, 1985), it is more probable that pHH502-1 lacks a $\operatorname{trf} A$-like gene. Since the $\operatorname{tr} f A$ gene is essential for a functional IncP replication system, the lack of this gene would imply that pHH502-1 possesses a replication system of another incompatibility group.

\section{Insensitivity to IncP incompatibility}

When the oriV region of RK2 is inserted into a high-copy-number vector replicon of another incompatibility group it exhibits strong incompatibility against IncP plasmids. Because the maintenance of the vector replicon is not affected by the presence of an IncP plasmid, this incompatibility is unidirectional, resulting in the preferential elimination of the IncP plasmid. We tested the effect of transformation with such a plasmid, pCT7, on resident pHH502-1 and observed $100 \%$ retention of $\mathrm{pHH} 502-1$. In contrast, similar experiments showed only $4 \%$ 
retention of pRK24 (a kanamycin-sensitive derivative of RK2) and complete loss of IncP $\beta$ plasmid R751, but $100 \%$ retention of each plasmid following transformation with pCT8, an otherwise isogenic plasmid lacking the oriV region (Thomas et al., 1980; Smith \& Thomas, 1985). This result confirmed that pHH502-1 possesses a replication system (of an unknown incompatibility group) which is not susceptible to the ori $V$-associated IncP determinants. This is consistent with the observation that transfer of pHH502-1 to a strain carrying RP4 results in the elimination of the resident plasmid from a high proportion of clones, while transfer of RP4 does not result in the elimination of resident pHH502-1, but gives unstable clones carrying both plasmids (Nugent et al., 1982).

\section{Presence of a korB-like gene}

Figurski et al. (1982) showed that a range of diverse IncP plasmids possess genes which can substitute for $\operatorname{kor} A$ and $\operatorname{kor} B$ in the control of the potentially host-lethal kil $A$ and $k i l B$ loci of RK2. We tested pHH502-1 for the ability to enable the establishment of compatible plasmids carrying the kilA and kilB loci of RK2. Following transformation of MV10(pHH502-1) with $k o r B$-dependent plasmid pCAS51, healthy colonies were obtained on medium selective for the incoming plasmid, indicating that pHH502-1 possesses a $k o r B$-like gene. However, transformation with korA-dependent plasmid pCAS54 gave equivocal results; no colonies were obtained from MV10, small slow-growing colonies from MV10(pHH502-1) and healthy colonies from MV10(pRK259.2), the positive control. It is unclear whether or not this result indicates that pHH502-1 possesses an active kor $A$-like gene.

\section{Evolutionary implications}

It is likely that pHH502-1 consists of a replicon of unknown incompatibility group carrying some part of an IncP replication system, presumably as a result of a replicon fusion followed by one or more deletion events. The IncP loci present include the ori $V$ region and the $k o r B$ gene, but not the $\operatorname{trf} A$ gene, essential for replication. This provides a clear, although trivial, explanation for the restricted host range of pHH502-1 compared to that of typical IncP plasmids. Since Nugent et al. (1982) found that the pili determined by pHH502-1 differ from those determined by other IncP plasmids serologically, morphologically and in the bacteriophage sensitivity which they confer, it is probable that the regions of the plasmid responsible for conjugal transfer did not form part of the IncP plasmid ancestor of pHH502-1.

Since $\mathrm{pHH} 502$, from which $\mathrm{pHH} 502-1$ is derived, also has a restricted host range it is probable that it also lacks a functional IncP replication system. The narrow host range of pMU700pMU707 and of pAV1 may also result from the lack of a complete functional IncP-like replication system.

The work described in this paper was funded and C. A. Smith is supported by MRC project grant G82/2421/3CB awarded to C. M. Thomas.

\section{REFERENCES}

Brown, N. L., Ford, S. J., Pridmore, R. D. \& Fritzinger, D. C. (1983). Nucleotide sequence of a gene from the Pseudomonas transposon Tn501 encoding mercuric reductase. Biochemistry 22, 40894095.

Chikami, G. K., Guiney, D. G., Schmidhauser, T. J. \& Helinski, D. R. (1985). Comparison of ten IncP plasmids: homology in the regions involved in plasmid replication. Journal of Bacteriology 162, 656660.

Coetzee, J. N., lecatsas, G. \& Coetzee, W. F. (1979). Properties of $R$ plasmid R772 and the corresponding pilus-specific phage PR772. Journal of General Microbiology 110, 263-273
Diver, W. P., Grinsted, J., Fritzinger, D. C., Brown, N. L., Altenbuchner, J., Rogowsky, P. \& SCHMIT, R. (1983). DNA sequences of and complementation by the $\operatorname{tnpR}$ genes of $\operatorname{Tn} 21, \mathrm{Tn} 501$ and Tn1721. Molecular and General Genetics 191, 189193.

Figurski, D. H., Pohlman, R. F., BechHofer, D. H., Prince, A. S. \& Kelton, C. A. (1982). The broad host range plasmid RK2 encodes multiple kil genes potentially lethal to Escherichia coli host cells. Proceedings of the National Academy of Sciences of the United States of America 79, 1935-1939.

Grant, A. J., Bird, P. I. \& Pittard, J. (1980). Naturally occuring plasmids exhibiting incompati- 
bility with members of incompatibility groups I and P. Journal of Bacteriology 144, 758-765.

Hedges, R. W., JACOB, A. E. \& SmIth, J. T. (1974). Properties of an $\mathbf{R}$ factor from Bordetella bronchiseptica. Journal of General Microbiology 84, 199-204.

HiNCHLIFFE, E. \& Vivian, A. (1980). Naturally occurring plasmids in Acinetobacter calcoaceticus: a $\mathbf{P}$ class $\mathbf{R}$ factor of restricted host range. Journal of General Microbiology 116, 75-80.

IngRam, L. C., Richmond, M. H. \& Sykes, R. B. (1973). Molecular characterization of the $\mathrm{R}$ factors implicated in the carbenicillin resistance of a sequence of Pseudomonas aeruginosa strains isolated from burns. Antimicrobial Agents and Chemotherapy 3, 279-288.

Jobanputra, R. S. \& DatTa, N. (1974). Trimethoprim $\mathbf{R}$ factors in enterobacteria from clinical specimens. Journal of Medical Microbiology 7, 169-177.

Kratz, J., Schmidt, F. \& Wiedemann, B. (1983). Characterization of $\operatorname{Tn} 2411$ and Tn2410, two transposons derived from R-plasmid R1767 and related to $\mathrm{Tn} 2603$ and $\mathrm{Tn} 21$. Journal of Bacteriology 155, 1333-1342.

Nugent, M. E., Ellis, K., Datta, N. \& Bradley, D. E. (1982). pHH502, a plasmid with IncP and IncI $\alpha$ characters, loses the latter by a specific recA-independent deletion event. Journal of General Microbiology 128, 2781-2790.

SMITH, C. A. \& Thomas, C. M. (1983). Deletion mapping of kil and kor functions in the $\operatorname{trf} A$ and $\operatorname{trf} B$ regions of broad host range plasmid RK2. Molecular and General Genetics 190, 245-254.
Smith, C. A. \& Thomas, C. M. (1985). Comparison of the nucleotide sequences of the vegetative origins of broad host range IncP plasmids R751 and RK2 reveals conserved features of probable functional importance. Nucleic Acids Research 13, 557-572.

SMITH, C. A. \& ThOMAs, C. M. (1987). Comparison of the organisation of the genomes of phenotypically diverse plasmids of incompatibility group $\mathbf{P}$ : members of the IncP $\beta$ sub-group are closely related. Molecular and General Genetics 206, 419-427.

Tanaka, M., Yamamoto, T. \& Sawal, T. (1983). Evolution of complex resistance transposons from an ancestral mercury transposon. Journal of Bacteriology 153, 1432-1438.

Thomas, C. M. \& Hussain, A. A. K. (1984). The korB gene of broad host range plasmid RK2 is a major copy number control element which may act together with $\operatorname{trf} B$ by limiting $\operatorname{trf} A$ expression. EMBO Journal 7, 1513-1519.

Thomas, C. M., Meyer, R. \& Helinski, D. R. (1980). Regions of broad-host-range plasmid $\mathrm{RK} 2$ which are essential for replication and maintenance. Journal of Bacteriology 141, 213-222.

Thomas, C. M., Smith, C. A., Shingler, V., Cross, M. A., Hussain, A. A. K. \& Pinkney, M. (1985). Regulation of replication and maintenance functions of broad host-range plasmid RK2. In Plasmids in Bacteria, pp. 261-276. Edited by D. B. Helinski, S. N. Cohen, D. B. Clewell, D. A. Jackson \& A. Hollaender. New York: Plenum Press. 